\title{
Fabrication and Characterization of $N \times N$ Plastic Optical Fiber Star Coupler based on Fused Combining
}

\author{
Kwang Taek Kim ${ }^{1 \dagger}$, Byeong Ha Lee ${ }^{2}$, Cherl-Hee Lee ${ }^{3}$, and Jonghun Lee ${ }^{3}$ \\ ${ }^{1}$ Department of Optoelectronics, Honam University, 59-1 Seobong-dong, Gwangsan-gu, Gwangju 505-714, Korea \\ ${ }^{2}$ School of Information and Communications, Gwangju Institute of Science and Technology, Gwangju 500-712, Korea \\ ${ }^{3}$ Robot System Division, Daegu Gyeongbuk Institute of Science \& Technology, Sangri 50-1, Hyeonpung-myeon, \\ Dalseong-gun, Daegu 711-813, Korea
}

(Received December 24, 2012; Revised manuscript February 4, 2013; Accepted February 4, 2013)

\begin{abstract}
High performance plastic optical fiber (POF) $N \times N$ star couplers are implemented based on fusing and combining technology. A set of cladding-removed POFs are fused into a solid body by heating and pressing them together to form the transition region between the input and output sides. The operation principle of the proposed star coupler is explained based on ray optics. To demonstrate the performance of the device, $2 \times 2,4 \times 4$ and $6 \times 6$ type POF couplers were fabricated and characterized. Performances of the POF star couplers were evaluated in terms of the flatness of the coupling ratios and excess losses.
\end{abstract}

Keywords: Plastic optical fiber, Fused combining, POF coupler, Automobile optical network, Ray optics OCIS codes: (080.2740) Geometric optical design; (060.2340) Fiber optics components; (060.1810) Buffers, couplers, routers, switches, and multiplexers

\section{INTRODUCTION}

For almost all applications of POF (plastic optical fiber) optical networks, proper couplers are required to split or combine various optical signals. Recently, POF networks have expanded their applications in automobiles, home networks, and automatic control and optical sensor systems. In particular, the optical network used in automobiles is evolving from a ring bus network into a star network as the demand for high speed data communication grows. ${ }^{[1]}$ There have been many approaches to the fabrication of POF couplers, such as side-polishing, ${ }^{[2,}{ }^{3]}$ twisting and fusing, ${ }^{[4,}$ ${ }^{5]}$ molding, ${ }^{[6]}$ mixing rods, ${ }^{[7]}$ using hollow taper waveguide, ${ }^{[8]}$ one dimensional layer cavity ${ }^{[9]}$ and polymer rectangular waveguide. ${ }^{[10]}$ High performance as well as simple fabrication for mass production is required for the POF couplers. The configurations of the POF couplers employing the molding, mixing rods, hollow taper waveguide, one layer cavity and polymer waveguide methods include intermediate media or air gaps. Therefore, undesired Fresnel reflection and optical excess loss may occur at interfaces. Though, the side-polishing and twisting and fusing method ${ }^{[3,4]}$ do not need the additional intermediate mediua, those methods demand time consuming and sophisticated processes such as side-polishing and fusing. Recently, we have reported a $4 \times 4$ type POF star coupler ${ }^{[12]}$ based on heating and fusing which shows high performance and mass productivity at the same time.

In this paper, we have examined feasibility of the fused combining technology for general $\mathrm{N} \times \mathrm{N}$ type of POF star couplers and we have reported performance of the devices. The three kinds of star couplers, $2 \times 2,4 \times 4$, and $6 \times 6$ POF star couplers are designed and characterized. In addition, a method to control the coupling ratio for $2 \times 2$ POF coupler is proposed and demonstrated. The cladding-removed parts of several POFs are combined into a single solid body by heating and pressing them together. In the fused and combined region, a number of optical rays are mixed and diffused, and then divided into the output channels with similar power distributions. The all in-line configuration of the proposing coupler obviates the need of additional polishing and connecting works in the fabrication process. Using ray optics, the characteristics of the POF couplers are simulated and predicted. The fabrication process is explained in detail and the performances of the POF star coupler are evaluated in terms of the flatness of the coupling ratios and excess losses.

${ }^{\dagger}$ E-mail: ktkim@honam.ac.kr

Color versions of one or more of the figures in this paper are available online. 


\section{DEVICE STRUCTURE AND OPERATION PRINCIPLE}

The structures of the proposing POF star couplers are shown in Fig. 1. Basically, several POFs are stacked together in a rectangular groove, and then fused into a single solid body by heating and pressing. In the transition regions, it is intended that each POF is adiabatically converted from the original circular shape into the shape of a square without change in the crosssection area. Therefore, there is no structural discontinuity along the longitudinal direction of the coupler, which reduces excess loss. The fused region is coated with a low refractive index epoxy to protect it from both mechanical damage and contamination. The optical beams in the coupling region are
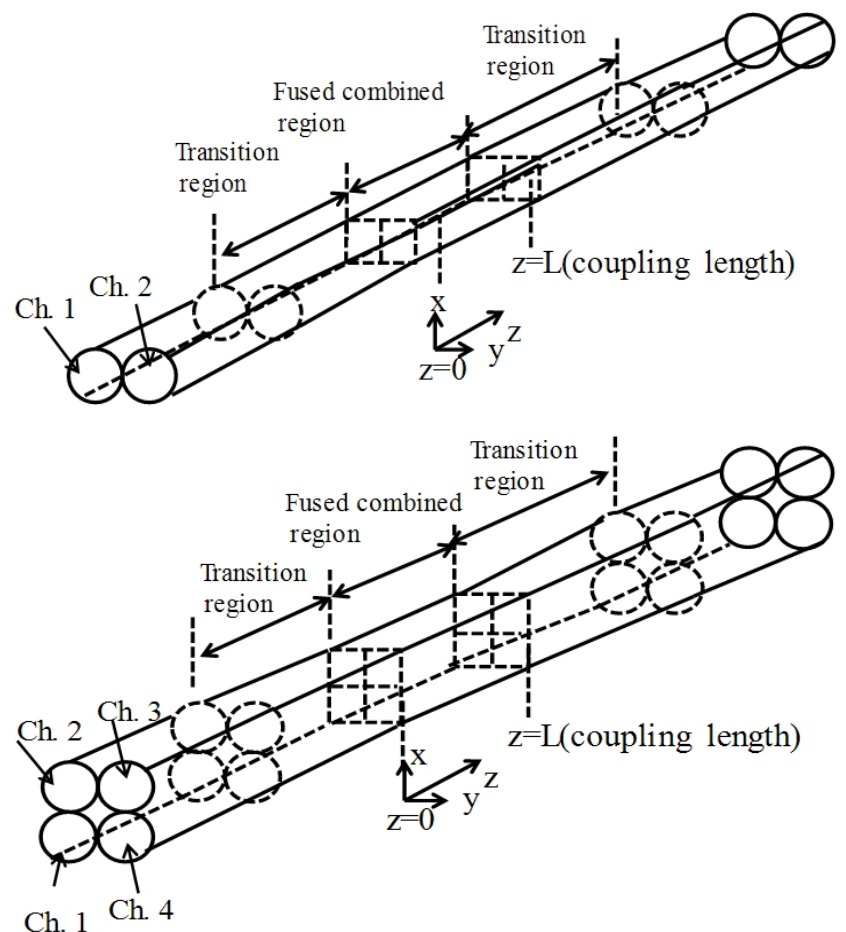

Ch. 1 Ch. 4

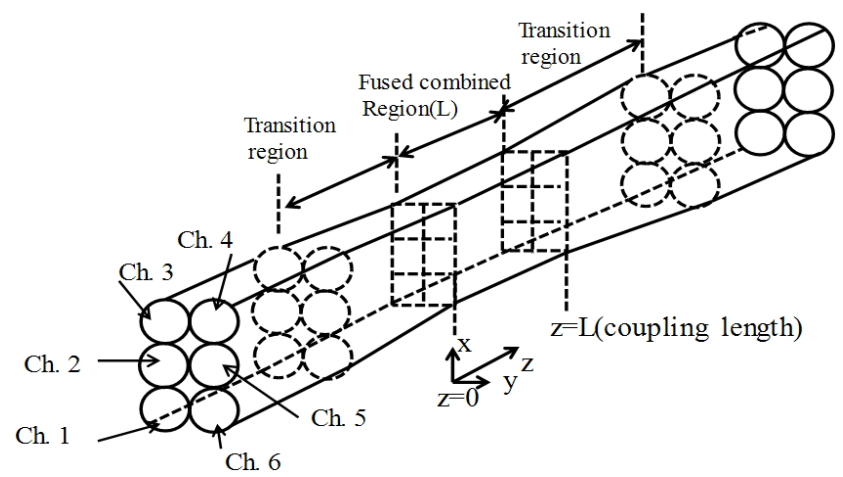

FIG. 1. Schematic structures of the proposed POF couplers, (a) $2 \times 2$ type POF coupler, (b) $4 \times 4$ type POF coupler, (c) $6 \times 6$ type POF coupler. guided by the TIR (total internal reflection) at the interface between the fused POF and its covering epoxy. As a result, if the coupling region is long enough, we can expect complete mingling of optical beams there and equal dividing into the output channels without any considerable loss.

The performance of a coupler is usually evaluated in terms of the excess loss and flatness of the coupling ratios. The optical coupling ratio from the $\mathrm{i}$-th input channel to the $\mathrm{j}$-th output channel and the associated excess loss are defined as:

$$
\text { Coupling } \cdot \text { ratio }=10 \log \frac{P O_{j}}{P I_{i}}
$$

$$
\text { Excess } \cdot \operatorname{loss}[d B]=-10 \log \frac{\sum_{j} P O_{j}}{P I_{i}}
$$

Where, $P I_{i}$ and $P O_{j}$ denote the input optical power at the $i$-th channel and the output optical power at the $j$-th channel, respectively. Flatness of coupling ratios means the difference between the maximum and minimum coupling ratios.

\section{NUMERICAL ANALYSIS}

The coupling ratio depends on the geometry of the coupling region and the distribution of the optical power at the input cross sectional plane, at $\mathrm{z}=0$. It has been theoretically and experimentally demonstrated that a rectangular solid body located between two bundles of POFs behaved as an optical ray mixer and optical power distributer. ${ }^{[10]}$ For the sake of simplicity, we assume that the width of the coupling region in the three proposing couplers is always $1.7 \mathrm{~mm}$ and that the heights of the $2 \times 2,4 \times 4$ and $6 \times 6$ type couplers are $0.85 \mathrm{~mm}, 1.7 \mathrm{~mm}$, and $2.55 \mathrm{~mm}$, respectively. It is also assumed that the optical power is homogenous across the cross section of an input port, and the shape of the input port is square at the $\mathrm{z}=0$ plane. All optical rays in the input port are uniformly distributed along the polar angle and the azimuthal angle.

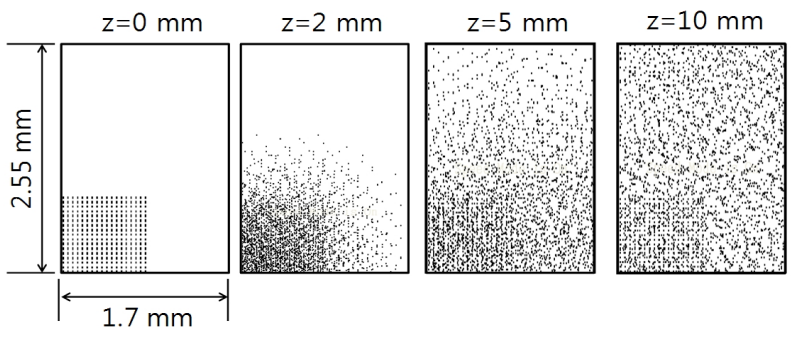

FIG. 2. Positions of rays along the $\mathrm{z}$ direction for the $6 \times 6$ type POF star coupler. 
Figure 2 simulates how the optical rays are mixed and diffused in the optical coupling region for the $6 \times 6$ type star coupler of Fig. 1(c). Channel 1 is assumed as the input channel. We can see that as the propagating distance increases the optical beam grows broader and becomes uniform. At the coupling length of $10 \mathrm{~mm}$, we can see a fairly uniform power distribution across the cross-section of the fused region. The behaviors of the other two types are similar to that of the $6 \times 6$ type star coupler.

Figure 3 shows the coupling ratios simulated in terms of the coupling length $(L)$ for the proposed three couplers. In all cases,
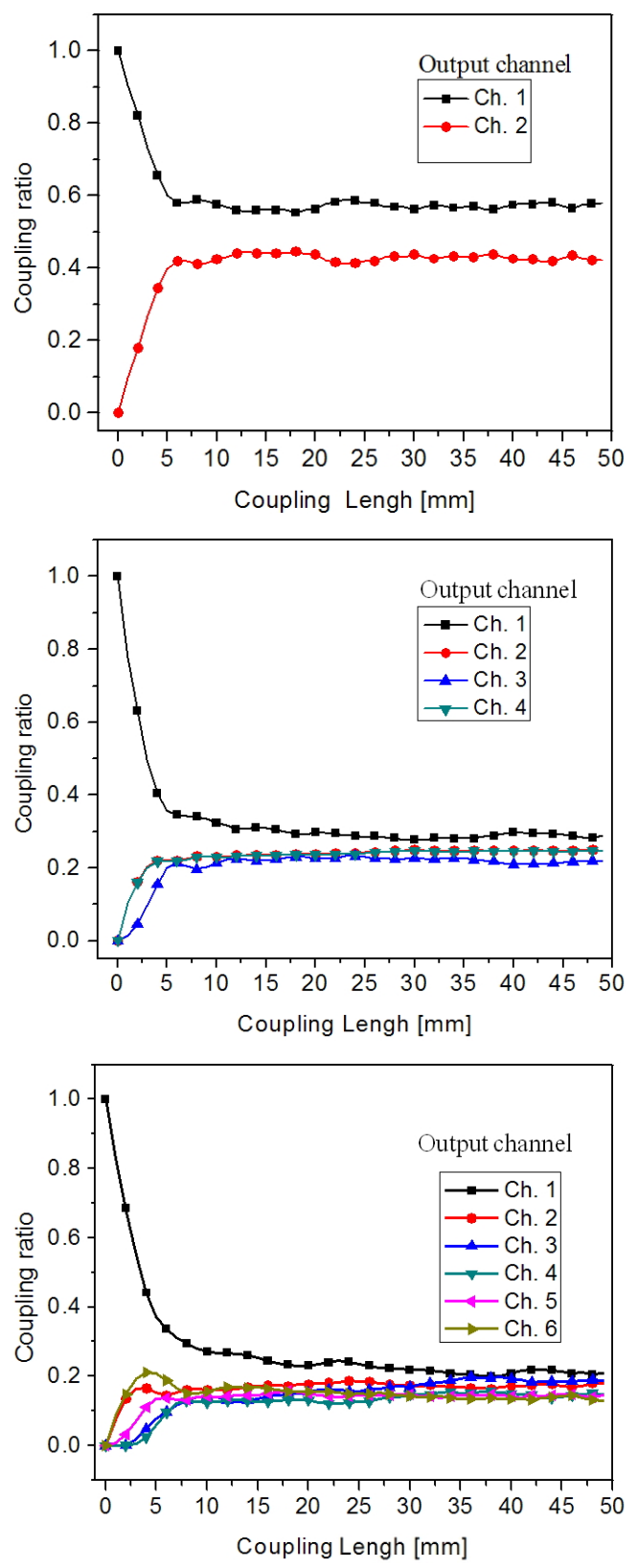

FIG. 3. Variation of coupling ratio with coupling length for input channel 1 , (a) $2 \times 2$ type POF coupler, (b) $4 \times 4$ type POF coupler, (c) $6 \times 6$ type $\mathrm{POF}$ coupler.
Ch. 1 is set as the input channel. For the $2 \times 2$ coupler, the coupling ratio to the Ch. 2 increases with the couplings, but is saturated at the length of $6 \mathrm{~mm}$ as shown with Fig. 3(a). Figure 3(b) shows that a similar thing happens with the $4 \times 4$ coupler. However, with the $6 \times 6$ coupler, the saturation happens near $\mathrm{L}=8 \mathrm{~mm}$. The interesting thing in all cases is that the self coupling to Ch. 1 is always higher than the cross couplings to other channels. Further, the self coupling ratio is not easily saturated compared with the cross coupling ratios. In fact, the coupling ratio depends on the launching condition of the optical source. As the divergence of the optical source decreases, thus the directivity of the optical source increases, the length of the fused region should be increased to retain the uniform coupling ratio.

\section{EXPERIMENT AND ANALYSIS}

The specifications of the POF used in the experiments were identical to the parameters used for the simulations. First, we prepared special jigs for fusing and combining several POFs at a time. The POFs were stacked in a carefully designed metal frame having a specially designed rectangular groove and pressed with a metal bar. The length and width of the groove was $20 \mathrm{~mm}$ and $1.7 \mathrm{~mm}$, respectively, as shown in Fig. 4. However, the depth of the groove was matched with the height of the stacked POFs. To make smooth transition region for the coupler, both ends of the groove were gradually widened in a funnel shape with a length of $10 \mathrm{~mm}$ each.

The surface of the groove of the base metal frame and the pressing metal bar were well polished to avoid optical scattering at the surface of the fused and combined region. The fabrication process of the proposing POF star coupler is explained in Fig. 4. At first, the cladding part of each POF was etched away using acetone. Typically, it took 30 minutes to etch a thickness of $10 \mu \mathrm{m}$

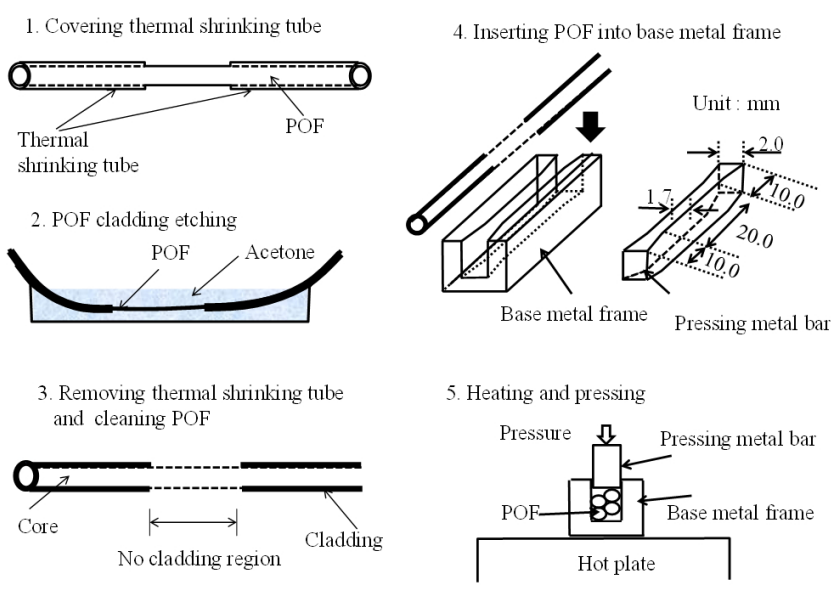

FIG. 4. Fabrication process of fused POF star couplers based on heating and pressing. 
of the cladding at room temperature. The length of the cladding to be etched was carefully adjusted, because the coupling of the input beam to several output POF channels occurs only at the region where the POF claddings were removed. A heat shrinking tube was used to protect the other part of the POF from unwanted etching. The cladding-etched POFs were inserted into the groove engraved along the base metal frame. The base metal frame was heated with a hot plate up to $120^{\circ} \mathrm{C}$. Using the pressing bar, the bundle of POFs was pressed together until they were fused into a single solid body. Since the POF cladding was very thin compared to its core diameter, no structural discontinuity was found along the fused and combined region. Finally, the fused and combined region was coated with an UV curable epoxy having a refractive index of 1.39. The three different types of POF couplers which were fabricated, are shown in Fig. 5 before epoxy coating. The heights of the fused and combined regions of the three couplers were $0.8 \mathrm{~mm}, 1.6 \mathrm{~mm}$ and $2.4 \mathrm{~mm}$, respectively, slightly smaller than the expected values.

A laser diode (LD) having a center wavelength of $635 \mathrm{~nm}$ was used as the optical source and launched into input channel 1 . We fabricated several $2 \times 2$ POF couplers with cladding etched regions of different lengths to examine the controllability of the coupling ratio. At first, the POF couplers made without the cladding etching was tested. The optical beams from the two output channels of the coupler are shown in Fig 6(a). No optical power coupling was observed and the excess loss was less than $0.2 \mathrm{~dB}$. From the experiment, we found two important facts. First, the cladding of POF still prevents optical coupling between the POFs even with the heating and pressing process. Second, the POF with transition regions at both ends transports the optical power without significant optical loss. Figure 6 shows that the optical power of output channel 2 increases with increasing the length of the cladding-removed region; (b) $5 \mathrm{~mm}$, (c) $10 \mathrm{~mm}$, and (d) $20 \mathrm{~mm}$. Therefore, it can be inferred that the

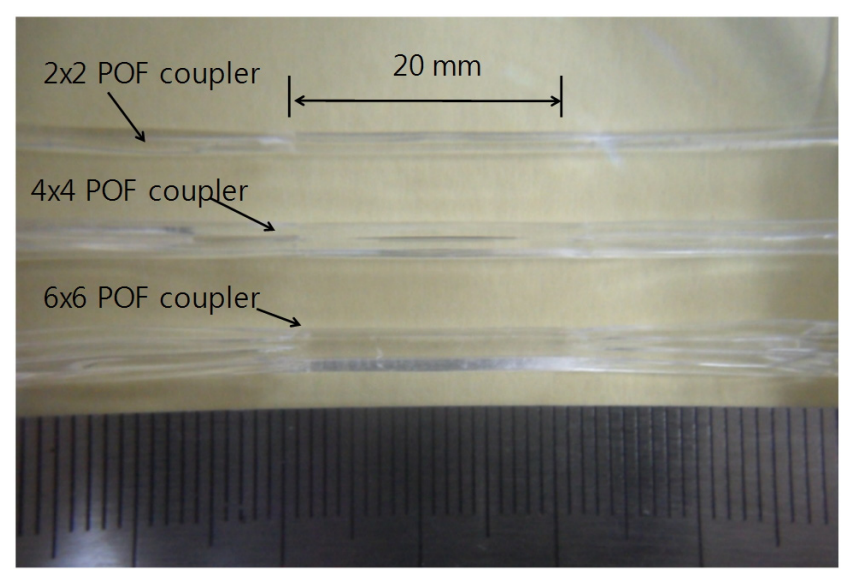

FIG. 5. Picture of the fabricated POF couplers length of the cladding-removed region is equal to the coupling length of the POF coupler. The measured coupling ratios and the excess losses are listed in Table. 1. The controllability of the coupling ratio is important for optical tap POF couplers employed for optical monitoring and optical sensors. ${ }^{[3,8]}$

We also examined the $4 \times 4$ type and the $6 \times 6$ type POF star couplers with the $20 \mathrm{~mm}$ cladding-removed regions. The measured coupling ratios, excess and flatness of coupling ratios for the fabricated $4 \times 4$ type and $6 \times 6$ type POF star couplers are listed in Table 2 and Table 3, respectively. The output powers of every output channel were measured while changing the input channels. The excess losses of both devices were less than $1.25 \mathrm{~dB}$ and 1.73 for $4 \times 4$ type and $6 \times 6$ type POF star couplers respectively, which is $1.0 \sim 1.5 \mathrm{~dB}$ smaller than the previously reported POF star couplers. ${ }^{[9,10]}$ We guess that this smaller loss was attributed to the transition regions. Due to the transition regions, there was no structural discontinuity along the longitudinal direction of the coupler. It resulted in smaller excess loss. In addition, it was
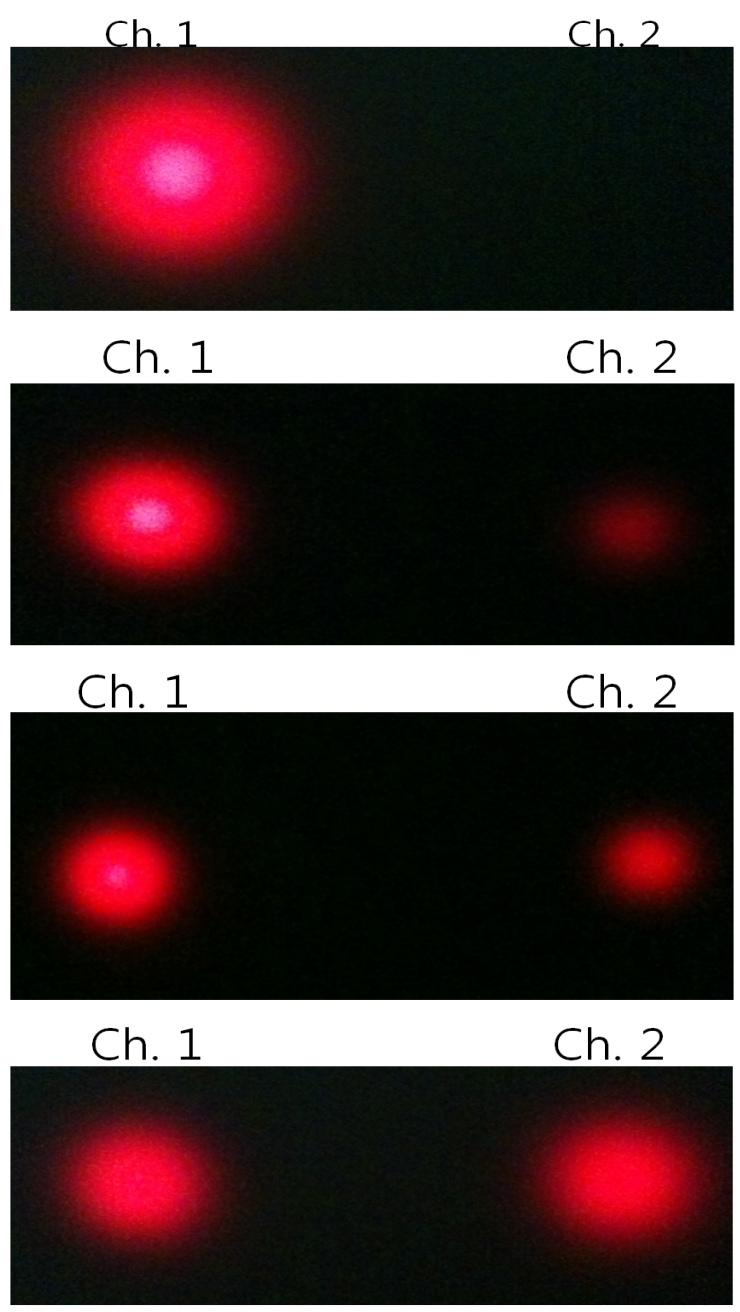

FIG. 6. Beam patterns of fabricated $2 \times 2$ type POF couplers with different coupling lengths (a) $0 \mathrm{~mm}$, (b) $5 \mathrm{~mm}$, (c) $10 \mathrm{~mm}$, (d) $20 \mathrm{~mm}$. 
TABLE 1. The measured coupling ratio and excess losses of the $2 \times 2$ type POF star couplers with different coupling lengths (L)

\begin{tabular}{|c|c|c|c|}
\hline $\begin{array}{c}\text { Coupling } \\
\text { length }[\mathrm{mm}]\end{array}$ & $\begin{array}{l}\text { Output } \\
\text { channel }\end{array}$ & $\begin{array}{l}\text { Coupling } \\
\text { ratio }[\mathrm{dB}]\end{array}$ & $\begin{array}{c}\text { Excess Loss } \\
{[\mathrm{dB}]}\end{array}$ \\
\hline \multirow{2}{*}{0} & Ch. 1 & - & \multirow{2}{*}{$<0.2$} \\
\hline & Ch. 2 & - & \\
\hline \multirow{2}{*}{5} & Ch. 1 & -1.1 & \multirow{2}{*}{$<1.0$} \\
\hline & Ch. 2 & -15.8 & \\
\hline \multirow{2}{*}{10} & Ch. 1 & -2.6 & \multirow{2}{*}{$<1.2$} \\
\hline & Ch. 2 & -6.6 & \\
\hline \multirow{2}{*}{20} & Ch. 1 & -3.4 & \multirow{2}{*}{$<1.0$} \\
\hline & Ch. 2 & -4.2 & \\
\hline
\end{tabular}

TABLE 2. The measured characteristics of the $4 \times 4$ type the POF star coupler with a coupling length(L) of $20 \mathrm{~mm}$

\begin{tabular}{|c|c|c|c|c|c|c|}
\hline \multirow{2}{*}{$\begin{array}{c}\text { Input } \\
\text { channel }\end{array}$} & \multicolumn{4}{|c|}{ Coupling ratio to output Channel } & \multirow{2}{*}{$\begin{array}{c}\text { Excess loss } \\
{[\mathrm{dB}]}\end{array}$} & \multirow{2}{*}{$\begin{array}{c}\text { Flatness of } \\
\text { coupling } \\
\text { ratio }[\mathrm{dB}]\end{array}$} \\
\hline & Ch. 1 & Ch. 2 & Ch. 3 & Ch. 5 & & \\
\hline Ch. 1 & -5.7 & -6.3 & -6.8 & -8.0 & 0.59 & 2.3 \\
\hline Ch. 2 & -7.2 & -5.1 & -8.9 & -6.9 & 0.79 & 3.8 \\
\hline Ch. 3 & -7.7 & -8.6 & -6.0 & -7.2 & 1.25 & 2.6 \\
\hline Ch. 4 & -9.1 & -7.2 & -7.3 & -5.7 & 1.14 & 3.4 \\
\hline
\end{tabular}

TABLE 3. The measured characteristics of the $6 \times 6$ type the POF star coupler with a coupling length(L) of $20 \mathrm{~mm}$

\begin{tabular}{|c|c|c|c|c|c|c|c|c|}
\hline \multirow{2}{*}{$\begin{array}{l}\text { Input } \\
\text { channel }\end{array}$} & \multicolumn{6}{|c|}{ Coupling ratio to output Channel } & \multirow{2}{*}{$\begin{array}{c}\text { Excess } \\
\text { loss } \\
{[\mathrm{dB}]}\end{array}$} & \multirow{2}{*}{$\begin{array}{c}\text { Flatness of } \\
\text { coupling } \\
\text { ratio }[\mathrm{dB}]\end{array}$} \\
\hline & Ch. 1 & Ch. 2 & Ch. 3 & Ch. 4 & Ch. 5 & Ch. 6 & & \\
\hline Ch. 1 & -9.3 & -8.9 & -9.6 & -9.8 & -9.2 & -9.5 & 1.59 & 0.9 \\
\hline Ch. 2 & -9.3 & -8.2 & -9.4 & -10.3 & -8.3 & -9.7 & 1.35 & 2.1 \\
\hline Ch. 3 & -9.7 & -8.1 & -8.3 & -10.5 & -8.7 & -8.0 & 1.01 & 2.2 \\
\hline Ch. 4 & -9.9 & -9.6 & -9.8 & -8.7 & -9.7 & -9.5 & 1.73 & 1.2 \\
\hline Ch. 5 & -9.8 & -9.0 & -9.6 & -9.6 & -8.4 & -9.1 & 1.44 & 1.4 \\
\hline Ch. 6 & -10.6 & -9.2 & -8.8 & -9.9 & -8.4 & -8.1 & 1.33 & 2.5 \\
\hline
\end{tabular}

found that the coupling region in the fused and combined solid body acts as both a good mixer and a distributer of optical rays. The flatness of coupling ratio for $4 \times 4$ type and $6 \times 6$ type POF star couplers, were less than $3.8 \mathrm{~dB}$ and $2.5 \mathrm{~dB}$, respectively.

Along with these high performances, the proposed POF couplers are fabricated with a simple fabrication process. It does not need additional processes such as cutting, polishing, and aligning due to their in-line configuration.

\section{CONCLUSION}

We proposed fused and combined type $2 \times 2,4 \times 4$ and $6 \times 6$ type
POF couplers manufactured by heating and pressing and demonstrated that they performed well enough for practical use. The cladding-removed POFs are combined into a solid body by pressing during heating. The fused and combined solid body operated as an effective optical power mixer and distributer. The coupling ratios of the $2 \times 2$ type POF coupler can be controlled by varying the coupling length, which is determined by the length of the cladding-removed region. The excess loss of the $2 \times 2,4 \times 4$ and $6 \times 6$ star couplers were less than $1.20 \mathrm{~dB}, 1.25 \mathrm{~dB}$ and $1.75 \mathrm{~dB}$, respectively. The flatness of coupling ratio for $2 \times 2$ type $4 \times 4$ type and $6 \times 6$ type POF star couplers, were less than $0.8 \mathrm{~dB}, 3.8 \mathrm{~dB}$ and $2.5 \mathrm{~dB}$, respectively. We anticipate that the proposed methods can be applied to the mass production of various high performance POF couplers.

\section{ACKNOWLEDGMENT}

This research was financially supported by the Ministry of Education, Science Technology (MEST) and this work was partially supported by the DGIST R\&D Program of the Ministry of Education, Science and Technology of Korea(12-RS-02).

\section{References}

1. T. Kibler, S. Poferl, G. Böck, H.-P. Huber, and E. Zeeb, “Optical data buses for automotive applications," J. Lightwave Technol. 22, 2184-2199 (2004).

2. D.-G. Kim, S. Y. Woo, D.-K. Kim, S.-H. Park, and J.-T. Hwang, "Fabrication and characteristics of plastic optical fiber directional couplers,” J. Opt. Soc. Korea 9, 99-102 (2005).

3. K. T. Kim, D. G. Kim, W. K. Hyun, K. B. Hong, K. G. Im, S. J. Baik, D. K. Kim, and H. Y. Hyun, "Side-coupled asymmetric plastic optical fiber coupler for optical sensor systems," J. Opt. Soc. Korea 12, 255-261 (2008).

4. Y. Jeong, S. Bae, and K. Oh, "All fiber $\mathrm{N} \times \mathrm{N}$ fused tapered plastic optical fiber (POF) power splitters for photodynamic therapy applicationsm," Current Applied Physics 9, 273-275 (2009).

5. M. S. A. Rahman, H. Guna, M. M. H. Harun, M. S. D. D. Zan, and K. Jumari, "Fabrication and characterization of customer 1x3 POF based optical coupler for home networking," IJCSNS International Journal of Computer Science and Network Security 8, 43-47 (2000).

6. Y. Takezawa, S. Akasaka, S. Ohara, T. Ishibashi, H. Asano, and N. Taketani, "Low excess losses in a Y-branching plastic optical waveguide formed through injection molding," Appl. Opt. 33, 2307-2312 (1994).

7. C. Yang, X. Sun, Y. Wang, M. Zhang, D. Ding, "1x7 plastic optical fiber coupler using cylindrical mixing rod," Proc. SPIE 4603, 183-187 (2001). 
8. A. A. Ehsan, S. Shaari, and M. K. A. Rahman, "Low cost 1x 2 Acrylic-based plastic optical fiber coupler with hollow taper waveguide," PIERS 2, 129-132 (2009).

9. K. Imoto, H. Sano, and M. Maeda, "Plastic optical fiber star coupler," Appl. Opt. 25, 3443-3447 (1986).
10. K. T. Kim, B. J. Han, M. K. Kim, Y. H. Kim, B. H. Lee, K. J. Cho, J. W. Kim, C.-H. Lee, and J. Lee, " $4 \mathrm{x} 4$ plastic optical fiber star coupler incorporated with a common polymer waveguide optical power distribution region," Fiber and Integrated Optics 30, 265-277 (2011). 